\title{
Improved Anti-Cancer Effect of Curcumin on Breast Cancer Cells by Increasing the Activity of Natural Killer Cells ${ }^{\mathbb{S}}$
}

\author{
Hwan Hee Lee ${ }^{1,2}$ and Hyosun Cho ${ }^{1,2 *}$ \\ ${ }^{1}$ College of Pharmacy, Duksung Women's University, Seoul 01369, Republic of Korea \\ ${ }^{2}$ Innovative Drug Center, Duksung Women's University, Seoul 01369, Republic of Korea
}

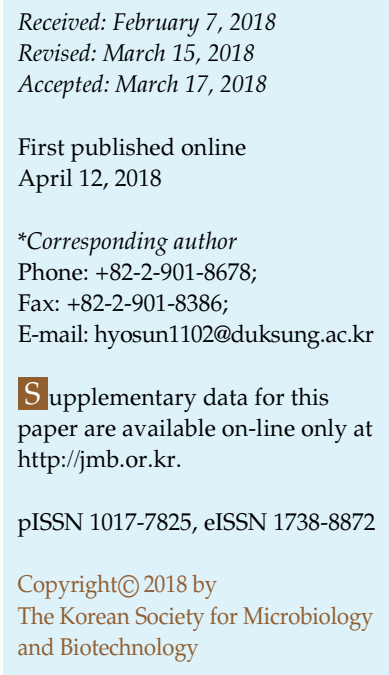

\begin{abstract}
Curcumin is known to possess various biological functions, including anti-inflammatory, antioxidative, and anti-cancer activities. Natural killer (NK) cells are large lymphocytes that directly kill cancer cells. However, many aggressive cancers, including breast cancer, were reported to escape the successful killing of NK cells in a tumor microenvironment. In this study, we investigated the anti-cancer effect of curcumin in coculture of human breast carcinoma MDA-MB-231 and NK (NK-92) cells. We found that curcumin had an immunestimulatory effect on NK-92 by increasing the surface expression of the CD16 ${ }^{+}$and CD56 ${ }^{\text {dim }}$ population of NK-92. We confirmed that the cytotoxic effect of NK-92 on MDA-MB-231 was significantly enhanced in the presence of curcumin, which was highly associated with the activation of Stat4 and Stat5 proteins in NK-92. Finally, this improved anticancer effect of curcumin was correlated with decreased expression of pErk and PI3K in MDA-MB-231.
\end{abstract}

Keywords: Curcumin, breast cancer, MDA-MB-231, natural killer cells

\section{Introduction}

Curcumin is one of the major food flavors that have a bright yellow color. Curcumin is extracted from the roots of the plant Curcuma longa and structurally is a hydrophobic polyphenol (1,7-bis(4-hydroxy-3-methoxyphenyl)-1,6heptadiene-3,5-dione) [1]. The biological activities of curcumin include anti-oxidant, anti-inflammatory, and anti-microbial effects [2-4]. From an anti-inflammatory perspective, curcumin has also shown to have anti-cancer effects on lung cancer, skin squamous cell carcinoma, pancreatic cancer, and breast cancer [5-8]. Jin et al. [5] found that curcumin treatment induced cell apoptosis via suppression of the PI3K/Akt pathway in A549 cells. Wu et al. [6] observed that curcumin decreased the invasive ability of A431 cells and also inhibited the transcription level of STAT3 mRNA in a dose-dependent manner. Curcumin was also reported to induce apoptosis by decreasing the Bcl-2/ Bax expression ratio and increasing caspase- $9 / 3$ activation in pancreatic cancer cells [7].

Breast cancer is the most common cancer among women and the fifth cause of death from cancer in the world [9]. Many studies have reported that curcumin has a strong inhibitory effect on the growth of various breast cancer cells in vitro as well as in vivo [10, 11]. The anti-cancer mechanisms of curcumin in breast cancer cells include modulating cell cycle regulation, inducing apoptosis, inhibiting tumor metastasis, and modifying the tumor immune environment [12].

Natural killer (NK) cells are large granular lymphoid cells that lyse cancer cells without prior activation [13, 14]. NK cells express two surface molecules, CD56 and CD16, which are closely associated with the major function of NK cells [15]. The $\mathrm{CD}^{+} 6^{+}$cells can be additionally divided into $\mathrm{CD}_{6} 6^{\mathrm{dim}}$ and $\mathrm{CD} 56^{\text {bright }}$. The $\mathrm{CD} 56^{\mathrm{dim}} \mathrm{CD} 16^{+}$population of NK cells is known to have more cytolytic granules (perforin, granzyme) than the $\mathrm{CD} 56^{\text {bright }} \mathrm{CD} 16^{+}$population and facilitates antibody-dependent cell cytotoxicity when recognizing tumor cells [15]. On the other hand, $\mathrm{CD} 56^{\text {bright }} \mathrm{CD} 16^{-/ \text {low }}$ cells secrete large amounts of cytokines such as IFN- $\gamma[16,17]$.

Recently, it has been shown that tumor cells or tumor microenvironments limit the anti-tumor activity of NK cells, 
which results in impairment of NK cell function [18-21]. Moreover, curcumin was reported to reverse the immunosuppression of NK cells and recover the cytotoxic effect on tumor cells. Bhaumik et al. [22] found that curcumin increased the production of nitric oxide of NK cells, which contributes to the tumoricidal effect of NK cells in AK-5 rat tumor. Fiala [23] showed that curcumin improved the cytotoxicity of NK cells, which increased apoptosis of the pancreatic cancer cells [23].

However, the molecular mechanism of curcumin in increasing the anti-cancer effect of NK cells is not clearly understood yet. In this study, we investigated the enhanced anti-cancer activity of NK cells by curcumin, especially against MDA-MB-231, which is an invasive ER negative human breast carcinoma. We further tried to identify the signaling molecules that are responsible for the anticancer activity of NK cells. Finally, we explored how this improved activity of antitumor NK cells affected MDA-MB-231.

\section{Materials and Methods}

\section{Specimen Preparation}

Curcumin $(>97.0 \%$ pure) was obtained from Tokyo Chemical Industry Co., Ltd. (Japan). Curcumin was dissolved using dimethyl sulfoxide (Sigma-Aldrich, USA) and diluted with each medium for human breast carcinoma MDA-MB-231 or natural killer (NK-92) cell treatment.

\section{Cell Lines and Culture}

Human breast carcinoma MDA-MB-231 and NK-92 cells were provided by ATCC. MDA-MB-231 was cultured in RPMI1640 (Corning, USA), supplemented with $10 \%$ heat-inactivated fetal bovine serum (FBS; Yong-In Frontier, Korea), and NK-92 was maintained in minimum essential Medium (MEM) alpha (Gibco, USA), supplemented with $20 \%$ FBS and $0.1 \mathrm{mM} \beta$-mercaptoethanol (Sigma-Aldrich, USA). Both cell lines were cultured in the presence of $100 \mathrm{U} / \mathrm{ml}$ penicillin and streptomycin (Gibco, USA) at $37^{\circ} \mathrm{C}$ in a humidified atmosphere with $5 \% \mathrm{CO}_{2}$.

\section{Cell Cytotoxicity of Curcumin on MDA-MB-231 and NK-92}

The cytotoxic effect of curcumin on MDA-MB-231 and NK-92 was assessed using thiazolyl blue tetrazolium bromide (MTT; SigmaAldrich, USA). Cells were seeded at a density of $1 \times 10^{4}$ cells per well in a 96-well flat-bottom plate, and serially diluted curcumin $(0,10,20,30,40,50,60,70,80,90$, or $100 \mu \mathrm{M})$ was added for 24 or $48 \mathrm{~h}$. Then, MTT solution was added to each well, and the were cells incubated in accordance with the reactive time of the solution. The absorbance was measured using a microplate reader (BMG Labtech, Germany) at $570 \mathrm{~nm}$.

\section{CytoTox 96 Nonradioactive Cytotoxicity Assay}

The specific cytotoxic effect of NK-92 on MDA-MB-231 was analyzed by a CytoTox 96 Non-Radioactive Cytotoxicity Assay kit (Promega, USA). Briefly, MDA-MB-231 was plated at a density of $5 \times 10^{3}$ cells per well in a 96-well flat-bottom plate and incubated overnight. Then, NK-92 was cocultured at an effector cell/target cell $(\mathrm{E} / \mathrm{T})$ ratio of 1:2 in the presence of different concentrations $(0$, $0.1,1$, or $10 \mu \mathrm{M}$ ) of curcumin for $5 \mathrm{~h}$. The cells were centrifuged and the supernatants were harvested and transferred to each well of a fresh plate. CytoTox 96 reagent was added to each well and incubated for $30 \mathrm{~min}$ at room temperature in the dark, and then the stop solution was added. The absorbance was recorded at $490 \mathrm{~nm}$ within $1 \mathrm{~h}$

\section{Flow Cytometry Analysis}

MDA-MB-231 was plated at a density of $5 \times 10^{5}$ cells per well in a 6-well plate overnight and cocultured with NK-92 $(\mathrm{E} / \mathrm{T}$ ratio $=$ $1: 2)$ in the presence of curcumin for $5 \mathrm{~h}$. NK-92 was harvested and stained with anti-CD56-APC (BD Biosciences, USA), anti-CD16-PE (BD Biosciences, USA), and VP (BD Biosciences, USA). The cells were analyzed by flow cytometry (Novocyte Flow Cytometer; ACEA Biosciences, USA). The positivity of CD56 or CD16 was determined by comparison with the defined cutoff values obtained with unstained control cells as previously described [24].

\section{ELISA}

MDA-MB-231 and NK-92 were cocultured in the presence of curcumin for $5 \mathrm{~h}$. Cell-free supernatants were harvested to measure the production of IFN- $\gamma$ using a human enzyme-linked immunosorbent assay kit (BD Biosciences, USA). Absorbance was measured at $450 \mathrm{~nm}$ using a microplate reader (BMG Labtech, Germany).

\section{Western Blot Analysis}

MDA-MB-231 and NK-92 were cocultured in the presence of curcumin for $5 \mathrm{~h}$. The cells were harvested differently according to each experiment. MDA-MB-231 or NK-92 was lysed by protein extraction buffer (Intron Biotechnology, Korea). The proteins in the cell lysates were quantified by the Bradford assay, separated by electrophoresis, and transferred to nitrocellulose membranes, which were then incubated with $1^{\text {st }}$ and $2^{\text {nd }}$ antibodies. PhosphoStat Antibody Sampler Kit (\#9914; Cell Signaling, USA) onto NK-92 and MAPK Family Antibody Sampler Kit (\#9926; Cell Signaling, USA) and Stat3 and pStat3 antibodies (Cell Signaling, USA) onto MDA-MB-231 were used for $1^{\text {st }}$ antibodies. The blots were visualized by enhanced chemiluminescent detection solutions (Intron Biotechnology, Korea).

\section{Statistical Analysis}

Data were processed using Microsoft Excel and results are presented as the mean $\pm \mathrm{SD}$. Comparisons of several means were performed by one-way or two-way analysis of variance followed by Fisher's exact test to identify significant differences between groups, with $p$-values of less than 0.05 being considered significant. 


\section{Results}

Cytotoxic Effect of Curcumin on MDA-MB-231 or NK-92

To confirm the cell cytotoxic effect of curcumin on human breast carcinoma MDA-MB-231, the cells were treated with serially diluted concentrations of curcumin for 24 or $48 \mathrm{~h}$, and cytotoxicity was validated by MTT assay. As expected, curcumin showed a great cytotoxic effect on MDA-MB-231 cells $\left(\mathrm{IC}_{50}=45 \mu \mathrm{M}\right.$ at $24 \mathrm{~h}, 23 \mu \mathrm{M}$ at $48 \mathrm{~h}$ ) (Fig. 1A, $p<0.0001$ ). However, curcumin did not affect the cell viability of NK-92 (Fig. 1B).

\section{Effect of Curcumin on the Surface Expression of CD16 or CD56 in NK-92}

To investigate whether curcumin has any effect on the activity of NK-92, NK-92 was cultured in the presence or absence of curcumin $(0,1$, or $10 \mu \mathrm{M})$ for $5 \mathrm{~h}$ and stained with fluorescent anti-CD56 and anti-CD16 antibodies. As mentioned in the Introduction, the expression of CD16 and CD56 in NK cells is directly related to cytolytic and maturation indicators, respectively, in NK cells. CD16 ${ }^{+}$ cells are more cytolytic than $\mathrm{CD} 16^{-}$cells and CD56 ${ }^{\text {dim }}$ cells are more mature than CD56 ${ }^{\text {bright }}$ cells [15]. Curcumin $(10 \mu \mathrm{M})$ increased the frequency of $\mathrm{CD}_{16}{ }^{+} \mathrm{NK}-92$ cells by up to $84.3 \%$ compared with untreated cells $(2.62 \%)$ and modified a substantial population of CD56 $6^{\text {bright }}$ NK-92 cells to become a CD56 ${ }^{\mathrm{dim}}$ population (curcumin-treated $41.8 \%$, untreated $27.3 \%$ ) (Figs. 2A and 2B). Furthermore, we examined whether curcumin would still maintain the stimulatory effect on NK-92 in a coculture with MDA-MB-231. The frequency of

A

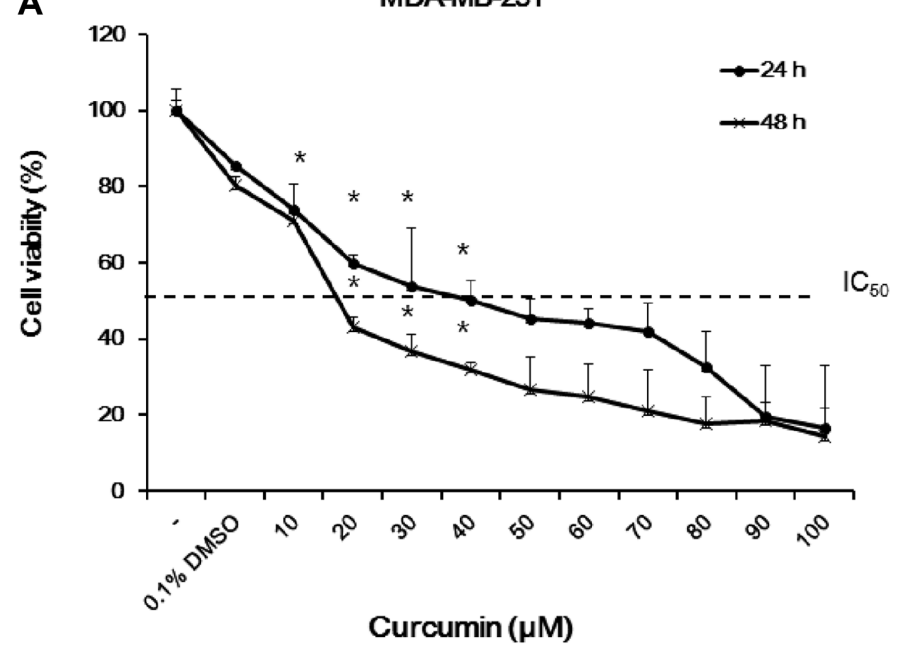

$\mathrm{CD}^{+} 6^{+} \mathrm{NK}-92$ in a coculture with MDA-MB-231 was dramatically increased from $1.54 \%$ to $56.1 \%$ in the presence of $10 \mu \mathrm{M}$ curcumin (Figs. 2C and 2D). Moreover, the frequency of the CD56 ${ }^{\mathrm{dim}} \mathrm{NK}-92$ population was increased from $37.4 \%$ to $42.7 \%$ when curcumin $(10 \mu \mathrm{M})$ was added in the coculture with MDA-MB-231.

\section{Effect of Curcumin on the Function of NK-92}

To clarify whether the increased frequency of $\mathrm{CD} 16^{+}$or $\mathrm{CD}^{\mathrm{dim}}{ }^{\mathrm{di}}$ in NK-92 was directly related to the functional activity of NK cells, first, the target cell cytotoxicity of NK92 was evaluated in coculture with MDA-MB-231. The effector (NK-92) and target (MDA-MB-231) ratio was 1:2 and different concentrations of curcumin $(0,0.1,1$, or $10 \mu \mathrm{M})$ were added in the culture (Fig. 3A). In the presence of curcumin, there was a significant improvement in NK cell cytotoxicity (Fig. 3A). In particular, $1 \mu \mathrm{M}$ of curcumin upregulated NK cell cytotoxicity with the maximum effect of $48.4 \%$ compared with the control $(24.7 \%)$. We also examined whether curcumin enhances the expression of IFN- $\gamma$, the major secreted cytokine of NK cells. Although coculture with MDA-MB-231 appeared to induce IFN- $\gamma$ production from NK-92 compared with NK-92 alone, curcumin treatment did not induce the secretion of IFN- $\gamma$ from NK-92 (Fig. 3B).

\section{Effect of Curcumin on the Expression of STAT Proteins in NK-92}

To determine how curcumin regulates the activity of NK cells at the molecular level, we assessed the expression of

Fig. 1. Cytotoxic effect of curcumin on MDA-MB-231 and NK-92.

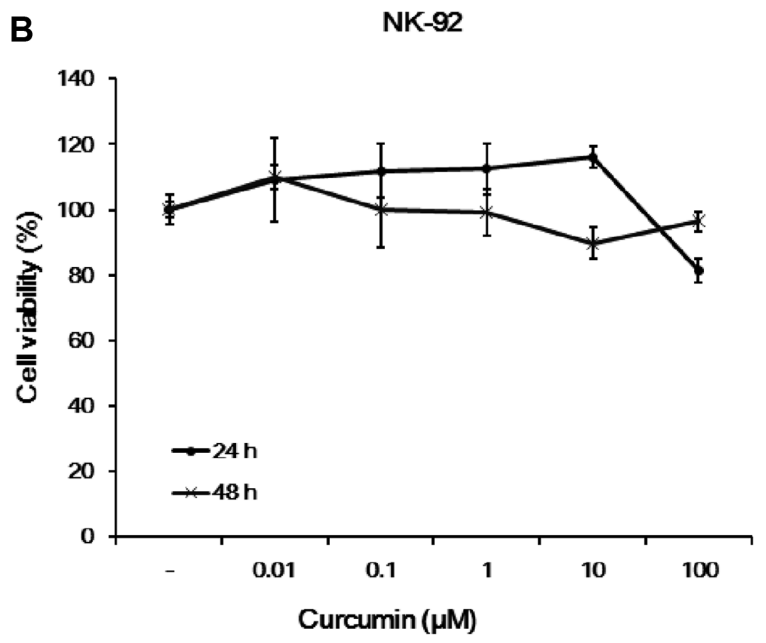

(A) MDA-MB-231 and (B) NK-92 were seeded at a density of $1 \times 10^{4}$ cells per well and serially diluted curcumin $(0,10,20,30,40,50,60,70,80,90$, or $100 \mu \mathrm{M}$ ) was added for 24 or $48 \mathrm{~h}$. Cytotoxicity was validated by MTT assay. 

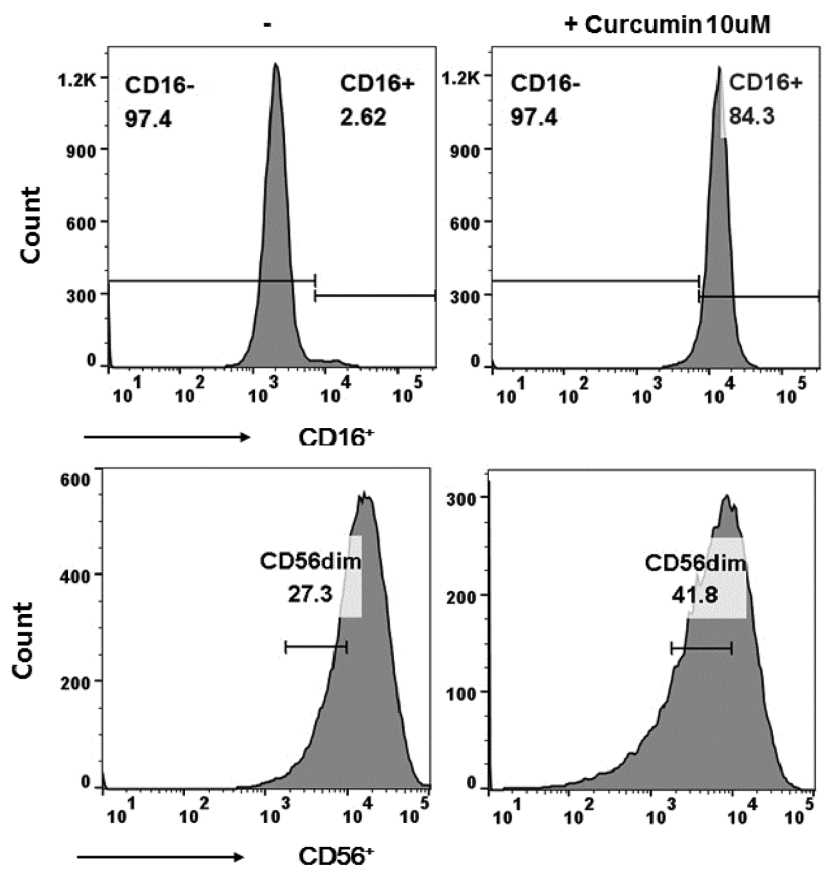

C
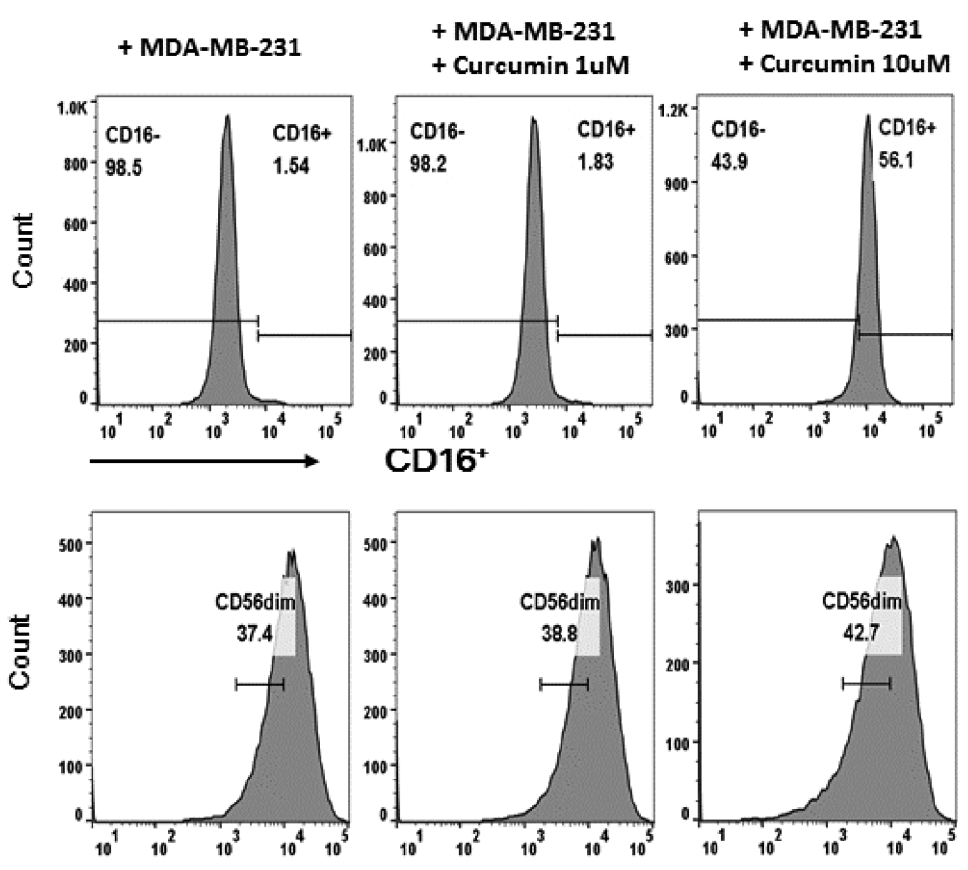

$\mathrm{CD}^{+} 6^{+}$
B
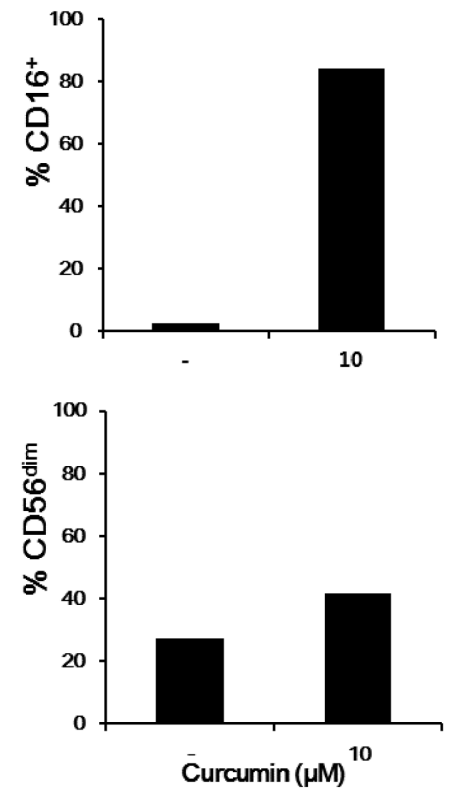

D
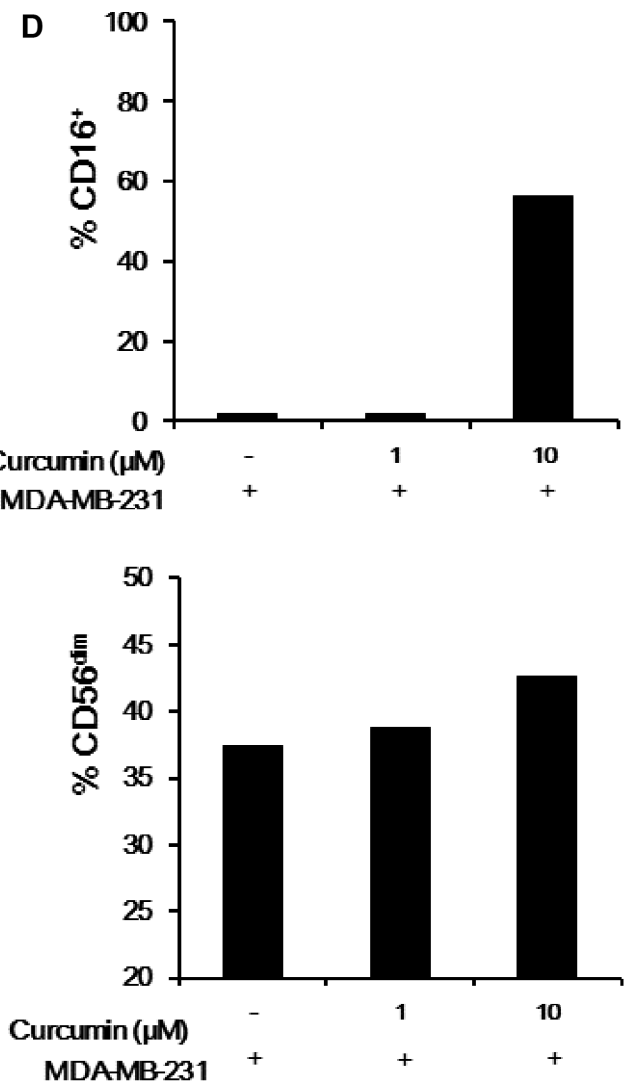

Fig. 2. Effect of curcumin on the surface expression of CD16 and CD56 in NK-92.

(A, B) NK-92 cells were cultured in the presence or absence of curcumin $(0,10 \mu \mathrm{M})$ or $($ C, D) cocultured with MDA-MB-231 in the presence or absence of curcumin $(0,1,10 \mu \mathrm{M})$ for $5 \mathrm{~h}$ and stained with fluorescent anti-CD56 and anti-CD16 antibodies. (A) Representative FACS plots showing CD16 and CD56 expression from NK-92 only or (C) from NK-92 cocultured with MDA-MB-231. 
A

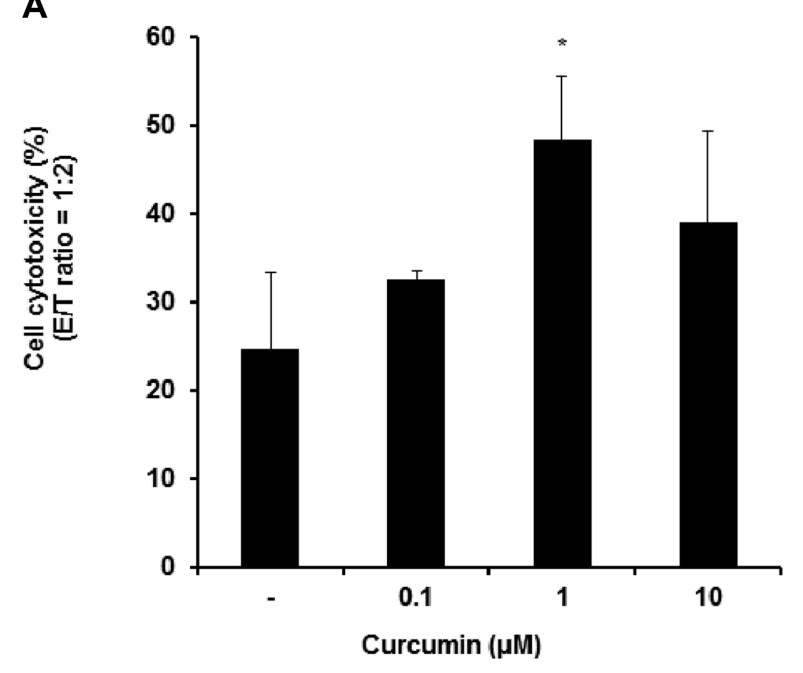

\begin{tabular}{|c|c|c|c|c|}
\hline & \multicolumn{4}{|c|}{ E/T ratio $=1: 2(\mathrm{NK}-92:$ MDA-MB-231) } \\
\hline $\begin{array}{c}\text { Curcumin } \\
(\mu \mathrm{M})\end{array}$ & - & $\mathbf{0 . 1}$ & $\mathbf{1}$ & $\mathbf{1 0}$ \\
\hline \hline value $(\%)$ & 24.7 & 32.6 & 48.4 & 39.1 \\
\hline
\end{tabular}

B

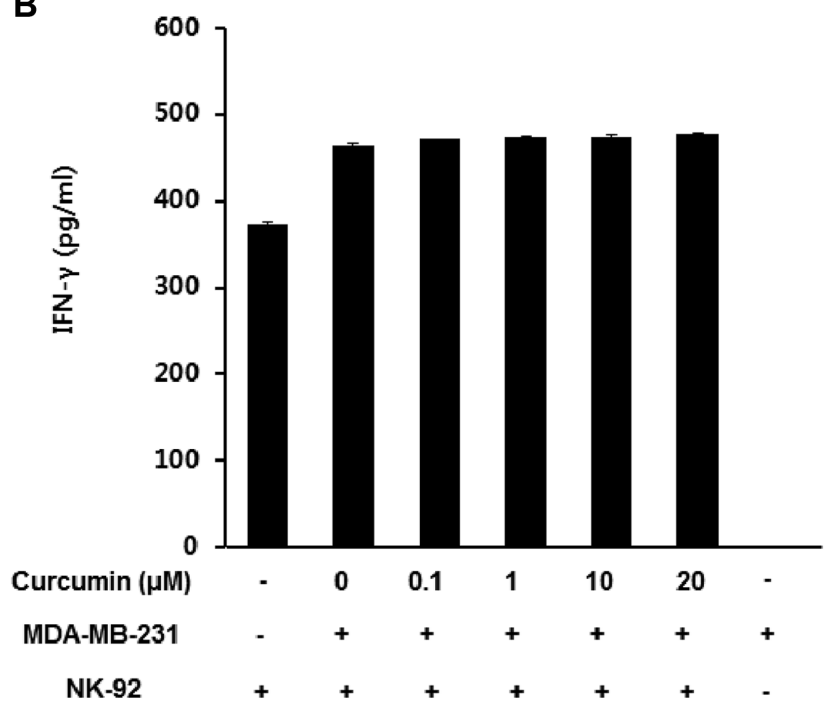

Fig. 3. Effect of curcumin on the function of NK-92.

MDA-MB-231 was plated at a density of $5 \times 10^{3}$ cells per well in a 96-well flat-bottom plate and incubated overnight. Then, NK-92 was cocultured at the E/T ratio of 1:2 in the presence of different concentrations $(0,0.1,1$, or $10 \mu \mathrm{M})$ of curcumin for $5 \mathrm{~h}$. (A) Percentages of cytotoxicity of NK-92 in the presence or absence of curcumin. (B) IFN- $\gamma$ production $(\mathrm{pg} / \mathrm{ml})$ in supernatants from coculture of NK-92 and MDA-MB-231 in the presence or absence of curcumin $(0,0.1,1,10$, or $20 \mu \mathrm{M})$.

signal transducer and activator of transcription (STAT) proteins from cell lysates of NK-92, which was cocultured with MDA-MB-231 in the presence or absence of curcumin. The STAT proteins are important transcription factors for NK cell activation and regulation. The expression levels of six different phosphorylated STAT proteins, pstat1, pstat2, pstat3, pstat4, pstat5, and pstat6 (Fig. 4A), show that curcumin increased the expression of pstat 4 and pstat5 proteins (Figs. 4A and 4B), which are known to be directly associated with the functional activation and maturation of NK cells.

Effect of Curcumin on the Expression of p38, pErk, Akt, PI3K, Stat3, and pStat3 Proteins in MDA-MB-231

The proliferation of cancer cells is known to be positively correlated with the activation of MAPK/ERK (p38, pErk) as well as PI3K/AKT (PI3K, Akt) pathway proteins [25]. Therefore, we examined whether curcumin inhibits the expression of p38, pErk, Akt, and PI3K proteins in MDAMB-231 cells when they are cocultured with NK-92. The expression of $\mathrm{pErk}$ and PI3K proteins from cell lysates of MDA-MB-231 showed a significant decrease in the presence of curcumin ( 0.1 and $1 \mu \mathrm{M})$ (Figs. 5A and 5B). Moreover, we also looked at whether curcumin modulates the expression of stat3, the major transcription factor for cancer metastasis [26], and the protein expression levels of stat 3 and pstat3 were not changed by curcumin (Figs. 5A and 5C).

\section{Discussion}

Initially, we confirmed the growth inhibitory effect of curcumin on human breast carcinoma MDA-MB-231, which agrees with many other studies [10, 19, 27]. Our data show that the $\mathrm{IC}_{50}$ values of curcumin after 24 and $48 \mathrm{~h}$ in MDAMB231 culture were 45 and $23 \mu \mathrm{M}$ (Fig. 1A), respectively, which is very similar to the values $(50 \mu \mathrm{M}$ at $24 \mathrm{~h}$ and $20 \mu \mathrm{M}$ at $48 \mathrm{~h}$ ) reported by Lv et al. [11]. Moreover, Liu et al. [10] reported that curcumin exerted an antiproliferative effect on MDA-MB-231 by inhibiting the expression of NF- $\mathrm{kB}$ and MMP-1. Chiu and Su [27] showed that curcumin even inhibited the cell migration of MDA-MB-231 by decreasing NF-kB p65 expression.

However, the effect of curcumin on immune cells, including NK cells, has not been well investigated even 
A

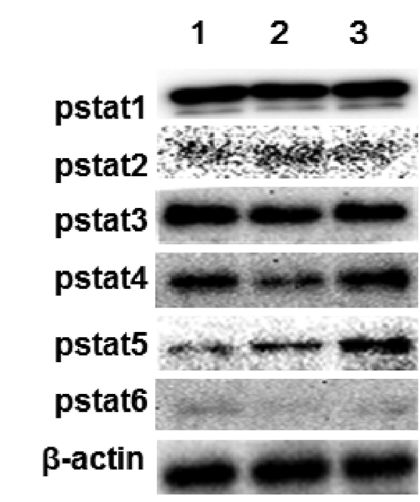

lane 1-NK-92(+MDA-MB-231)

lane 2-NK-92 (+MDA-MB-231 + Curcumin $0.1 \mu \mathrm{M}$ ) lane 3-NK-92 (+MDA-MB-231 + Curcumin $1 \mu \mathrm{M}$ )
B

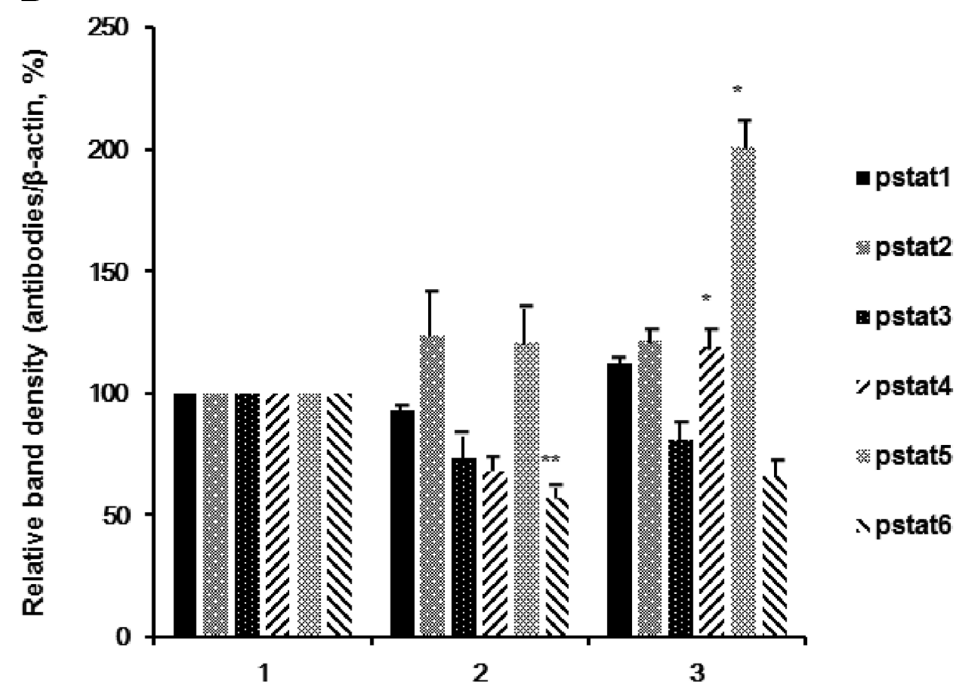

Fig. 4. Effect of curcumin on the expression of STAT proteins in NK-92.

NK-92 and MDA-MB-231 were cocultured in the presence or absence of curcumin $(0,0.1$, or $1 \mu \mathrm{M})$ for $5 \mathrm{~h}$. Cell lysates from NK-92 were assessed for the expression of pstat proteins. $\beta$-Actin was used as the loading control. (A) pstat1, pstat2, pstat3, pstat4, pstat5, and pstat6 protein expressions. (B) Relative band intensity of the pstats compared with the loading control.

though a variety of immune cells are critical components in the tumor microenvironment that contribute to tumor growth as well as metastasis [28]. Only a few studies have reported on the immunomodulatory effect of curcumin on NK cells. Zhang et al. [29] reported that curcumin was able to partly rescue tumor exosome-mediated inhibition of NK cell activation in mouse breast cancer. Fiala [23] found that curcumin-enriched compound enhanced NK cell-induced apoptosis of pancreatic cancer cells. A recent study showed that a curcumin-enriched mixture induced the activation of NK cells and phagocytosis of macrophages [30]. However, these studies did not explore the fundamental molecular mechanisms of NK cell activation by curcumin.

In this study, we observed that curcumin treatment directly activated the cytolytic activity of NK-92 against human breast carcinoma MDA-MB-231 (Fig. 3), which strongly correlated with the increased frequency of $\mathrm{CD}^{+} 6^{+}$ NK-92 cells (Fig. 2). Therefore, our results provide solid evidence for a direct immune-stimulatory effect of curcumin on NK cells. Curcumin did not influence the production of IFN- $\gamma$ from NK-92 in coculture with MDA-MB-231, which disagrees with a report from another study [23]. Fiala [23] showed that curcumin actually inhibited IFN- $\gamma$ production by blocking stat1 signaling in NK cells cocultured with human pancreatic cancer cells. We speculate that there is a difference in complicated carcinogenetic mechanisms between breast cancer cells and pancreatic cancer cells. The Janus kinase (JAK)-STAT pathway contributes to cytokine signaling in several tumor cells and also to the activation or inhibition of NK cell functions. In particular, in the JAKSTAT pathway, phosphorylation of stat1, stat4, and stat5 can stimulate the maturation and cytotoxicity of NK cells, whereas stat3 sand stat6 can restrain NK cell activity [31]. Our data show that the molecular mechanism responsible for the activation of NK-92 by curcumin is specifically associated with the upregulation of stat4 and stat5 (Fig. 4). To the best of our knowledge, our study is the first report that shows the direct effect of curcumin on the expression of STAT proteins in NK cells.

Finally, the curcumin-induced immunostimulatory effect of NK cells resulted in significant cell cytotoxicity on MDAMB-231 in $1 \mu \mathrm{M}$ of curcumin compared with untreated cells (Fig. 3A). Lower concentrations of curcumin $(0.1$ and $1 \mu \mathrm{M})$ did not exert a cytotoxic effect on MDA-MB-231 (Fig. S1), whereas $10 \mu \mathrm{M}$ of curcumin showed a slight effect on the cell viability of MDA-MB-231 (Fig. 1A). Therefore, the dramatic cytotoxic effect on MDA-MB-231 of curcumin (Fig. 3A) certainly resulted from the activation of NK-92 by curcumin treatment. Our data suggest that the anticancer effect of curcumin at relatively low concentrations (0.1 and $1 \mu \mathrm{M})$ that have hardly any influence on the cell viability of MDA-MB-231 is synergistically improved by the enhanced 
A

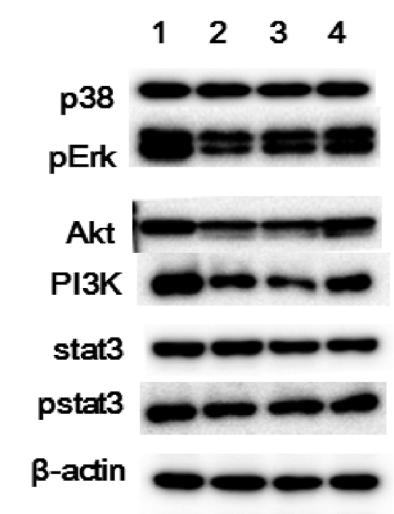

lane 1-MDA-MB-231 (+ NK-92)

lane 2-MDA-MB-231 (+ NK-92 + Curcumin $0.1 \mu \mathrm{M})$

lane 3-MDA-MB-231 (+ NK-92 + Curcumin $1 \mu \mathrm{M})$

lane 4-MDA-MB-231 (+ NK-92 + Curcumin $10 \mu \mathrm{M})$
B

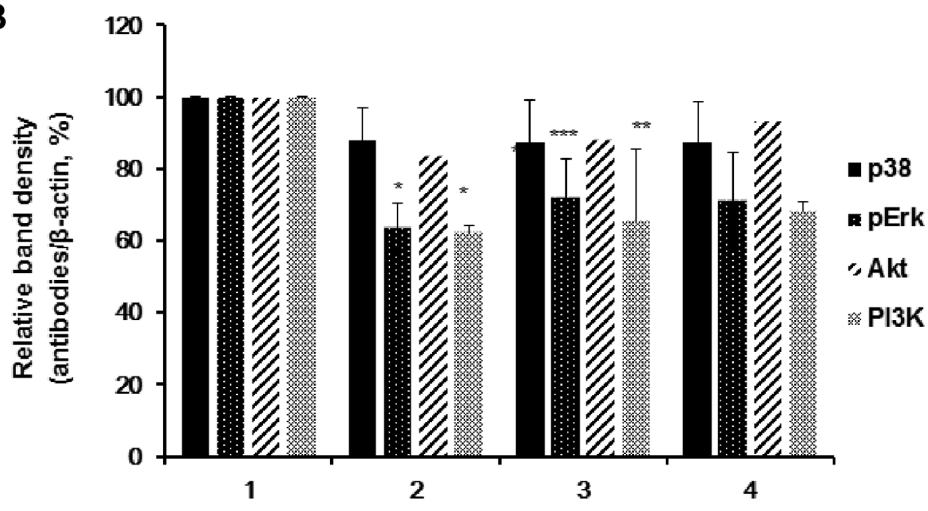

C

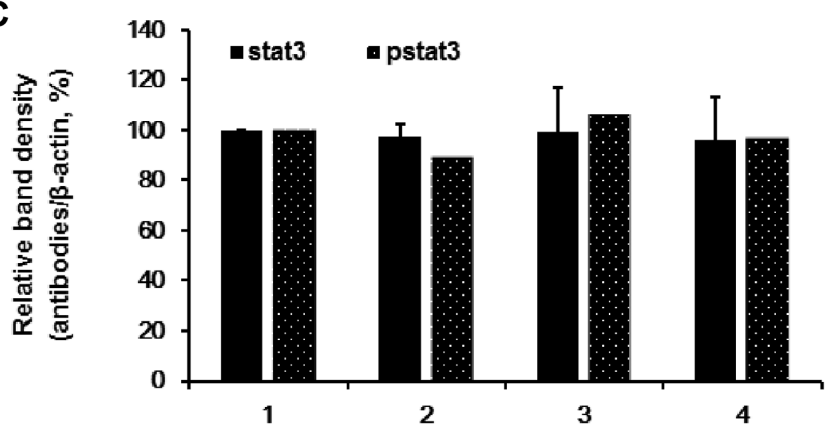

Fig. 5. Effect of curcumin on the expression of p38, pErk, AKT, PI3K, stat3, and pstat3 proteins in MDA-MB-231.

MDA-MB-231 and NK-92 were co-cultured in presence or absence of curcumin $(0,0.1$, or $1 \mu \mathrm{M})$ for $5 \mathrm{~h}$. Cell lysates from MDA-MB-231 were assessed for the expression of p38, pErk, Akt, PI3K, stat3, and pstat3 proteins. $\beta$-Actin was used as the loading control. (A) p38, pErk, Akt, PI3K, stat3, and pstat3 protein expression. (B) Relative band intensity of p38, pErk, Akt, PI3K, stat3, and pstat3 compared with the loading control.

antitumor activity of NK-92. This tumor cytotoxic event was shown to be highly associated with downregulation of pErk and PI3K expression, but not of p38 expression (Fig. 5). The p38/Erk pathway is known to play a primary role in integrating external signals into intracellular signals to promote cell growth and proliferation in many cancer cells. The PI3K and Akt proteins also participate in another critical intracellular signal transduction pathway in cell cycle regulation [25]. NF- $\mathrm{KB}$ is an essential transcription factor that controls cell proliferation and survival. The activation of NF- $\mathrm{\kappa B}$ can be induced by multiple intracellular signaling mechanisms, including the p38/Erk and PI3K/Akt pathways, and persistent activation of NF- $\mathrm{\kappa B}$ was observed in various cancers. Thus, NF-kB-associated signaling proteins, including p38, Erk, PI3K, and Akt, have become selective candidates for anti-cancer therapeutic targeting [32]. Recent studies reported that curcumin can directly downregulate the expression of pErk, Akt, and PI3K proteins in MDA-MB 231. However, those groups used much higher concentrations of curcumin $(25-50 \mu \mathrm{M})$ than used in our study to present a considerable reduction in the expression of Akt and PI3K signaling molecules [33, 34]. We used only $0.1 \mu \mathrm{M}$ of curcumin in a coculture of MDAMB 231 and NK-92 to achieve a significant decrease in pErk and PI3K expression, which suggests a synergistic effect of curcumin itself on MDA-MB-231 and through the activation of NK cells.

Additionally, we investigated whether the expression of stat3 protein in MDA-MB-231 is changed in coculture with NK-92 by curcumin treatment. Stat 3 protein is known to be constitutively activated in some cancer cells, which leads to promotion of tumor growth and metastasis [26]. Therefore, we tried to investigate whether or not the synergistic effect of curcumin contributes to tumor metastasis. Sui et al. [35] reported that the antitumor effect of NK cells is involved in the inhibition of hepatocellular carcinoma, which is mediated by a blockade of stat 3 in hepatocellular carcinoma. However, we were not able to observe any modulation in 
the expression of stat3 or pstat3 in MDA-MB-231 by the curcumin-induced antitumor activity of NK cells (Figs. 5A and 5C).

In summary, our study presented that the cytotoxic activity of NK cells against MDA-MB-231 was positively upgraded by curcumin. Moreover, the activation of stat 4 and stat5 molecules seems to be responsible for the enhanced anti-cancer activity of the NK cells, which led to the downregulation of pErk and PI3K expression in MDAMB-231.

\section{Acknowledgments}

This research was supported by Duksung Women's University Research Grants (2017-3000002825). This work was also supported by the Priority Research Center Program through the NRF funded by the Korean Ministry of Education, Science and Technology (2016R1A6A1A03007648).

\section{Conflict of Interest}

The authors have no financial conflicts of interest to declare.

\section{References}

1. Sinha D, Biswas J, Sung B, Aggarwal BB, Bishayee A. 2012. Chemopreventive and chemotherapeutic potential of curcumin in breast cancer. Curr. Drug Targets 13: 1799-1819.

2. Bengmark S, Mesa MD, Gil A. 2009. Plant-derived health: the effects of turmeric and curcuminoids. Nutr. Hosp. 24: 273-281.

3. Jurenka JS. 2009. Anti-inflammatory properties of curcumin, a major constituent of Curcuma longa: a review of preclinical and clinical research. Altern. Med. Rev. 14: 141-153.

4. Hussain Z, Thu HE, Amjad MW, Hussain F, Ahmed TA, Khan S. 2017. Exploring recent developments to improve antioxidant, anti-inflammatory and antimicrobial efficacy of curcumin: a sreview of new trends and future perspectives. Mater. Sci. Eng. C Mater. Biol. Appl. 77: 1316-1326.

5. Jin H, Qiao F, Wang Y, Xu Y, Shang Y. 2015. Curcumin inhibits cell proliferation and induces apoptosis of human non-small cell lung cancer cells through the upregulation of miR-192-5p and suppression of PI3K/Akt signaling pathway. Oncol. Rep. 34: 2782-2789.

6. Wu J, Lu WY, Cui LL. 2015. Inhibitory effect of curcumin on invasion of skin squamous cell carcinoma A431 cells. Asian Pac. J. Cancer Prev. 16: 2813-2818.

7. Zhao Z, Li C, Xi H, Gao Y, Xu D. 2015. Curcumin induces apoptosis in pancreatic cancer cells through the induction of forkhead box $\mathrm{O} 1$ and inhibition of the PI3K/Akt pathway.
Mol. Med. Rep. 12: 5415-5422.

8. Liu Y, Zhou J, Hu Y, Wang J, Yuan C. 2017. Curcumin inhibits growth of human breast cancer cells through demethylation of DLC1 promoter. Mol. Cell. Biochem. 425: 47-58.

9. Torre LA, Bray F, Siegel RL, Ferlay J, Lortet-Tieulent J, Jemal A. 2015. Global cancer statistics. CA Cancer J. Clin. 65: 87-108.

10. Liu Q, Loo WT, Sze SC, Tong Y. 2009. Curcumin inhibits cell proliferation of MDA-MB-231 and BT-483 breast cancer cells mediated by down-regulation of NFkappaB, cyclinD and MMP-1 transcription. Phytomedicine 16: 916-922.

11. Lv ZD, Liu XP, Zhao WJ, Dong Q, Li FN, Wang HB, et al. 2014. Curcumin induces apoptosis in breast cancer cells and inhibits tumor growth in vitro and in vivo. Int. J. Clin. Exp. Pathol. 7: 2818-2824.

12. Banik U, Parasuraman S, Adhikary AK, Othman NH. 2017. Curcumin: the spicy modulator of breast carcinogenesis. J. Exp. Clin. Cancer Res. 36: 98.

13. Doherty DG, O'Farrelly C. 2000. Innate and adaptive lymphoid cells in the human liver. Immunol. Rev. 174: 5-20.

14. Joyce JA, Pollard JW. 2009. Microenvironmental regulation of metastasis. Nat. Rev. Cancer 9: 239-252.

15. Cooper MA, Fehniger TA, Caligiuri MA. 2001. The biology of human natural killer-cell subsets. Trends Immunol. 22: 633-640.

16. Ferlazzo G, Thomas D, Lin SL, Goodman K, Morandi B, Muller WA, et al. 2004. The abundant NK cells in human secondary lymphoid tissues require activation to express killer cell Ig-like receptors and become cytolytic. J. Immunol. 172: $1455-1462$

17. Romagnani C, Juelke K, Falco M, Morandi B, D'Agostino A, Costa R, et al. 2007. CD56brightCD16- killer Ig-like receptorNK cells display longer telomeres and acquire features of CD56dim NK cells upon activation. J. Immunol. 178: 4947-4955.

18. Castriconi R, Cantoni C, Della Chiesa M, Vitale M, Marcenaro E, Conte R, et al. 2003. Transforming growth factor beta 1 inhibits expression of NKp30 and NKG2D receptors: consequences for the NK-mediated killing of dendritic cells. Proc. Natl. Acad. Sci. USA 100: 4120-4125.

19. Liu $Q$, Sun $Y$, Rihn S, Nolting A, Tsoukas PN, Jost $S$, et al. 2009. Matrix metalloprotease inhibitors restore impaired NK cell-mediated antibody-dependent cellular cytotoxicity in human immunodeficiency virus type 1 infection. J. Virol. 83: 8705-8712.

20. Hoechst B, Voigtlaender T, Ormandy L, Gamrekelashvili J, Zhao F, Wedemeyer H, et al. 2009. Myeloid derived suppressor cells inhibit natural killer cells in patients with hepatocellular carcinoma via the NKp30 receptor. Hepatology 50: 799-807.

21. Balsamo M, Scordamaglia F, Pietra G, Manzini C, Cantoni C, Boitano $\mathrm{M}$, et al. 2009. Melanoma-associated fibroblasts modulate NK cell phenotype and antitumor cytotoxicity. Proc. Natl. Acad. Sci. USA 106: 20847-20852.

22. Bhaumik S, Jyothi MD, Khar A. 2000. Differential modulation 
of nitric oxide production by curcumin in host macrophages and NK cells. FEBS Lett. 483: 78-82.

23. Fiala M. 2015. Curcumin and omega-3 fatty acids enhance NK cell-induced apoptosis of pancreatic cancer cells but curcumin inhibits interferon-gamma production: benefits of omega-3 with curcumin against cancer. Molecules 20: 3020-3026.

24. Lee HH, Kang H, Cho H. 2017. Recovery of NK(CD56+CD3-) cells after one year of tenofovir therapy for chronic hepatitis B infection. J. Microbiol. Biotechnol. 27: 1204-1308.

25. Corno C, Gatti L, Lanzi C, Zaffaroni N, Colombo D, Perego P. 2016. Role of the receptor tyrosine kinase Axl and its targeting in cancer cells. Curr. Med. Chem. 23: 1496-1512.

26. Devarajan E, Huang S. 2009. STAT3 as a central regulator of tumor metastases. Curr. Mol. Med. 9: 626-633.

27. Chiu TL, Su CC. 2009. Curcumin inhibits proliferation and migration by increasing the $\mathrm{Bax}$ to $\mathrm{Bcl}-2$ ratio and decreasing NF-kappaBp65 expression in breast cancer MDA-MB-231 cells. Int. J. Mol. Med. 23: 469-475.

28. Lee HH, Kang H, Cho H. 2017. Natural killer cells and tumor metastasis. Arch. Pharm. Res. 40: 1037-1049.

29. Zhang HG, Kim H, Liu C, Yu S, Wang J, Grizzle WE, et al. 2007. Curcumin reverses breast tumor exosomes mediated immune suppression of NK cell tumor cytotoxicity. Biochim. Biophys. Acta 1773: 1116-1123.

30. Trivedi MK, Mondal SC, Gangwar M, Jana S. 2017. Immunomodulatory potential of nanocurcumin-based formulation. Inflammopharmacology 25: 609-619.

31. Gottardt D, Sexl V. 2017. STATs in NK-cells: the good, the bad, and the ugly. Front. Immunol. 7: 694.

32. Pires BRB, Silva R, Ferreira GM, Abdelhay E. 2018. NFkappaB: two sides of the same coin. Genes (Basel) 9: E24.

33. Guan F, Ding Y, Zhang Y, Zhou Y, Li M, Wang C. 2016. Curcumin suppresses proliferation and migration of MDAMB-231 breast cancer cells through autophagy-dependent Akt degradation. PLoS One 11: e0146553.

34. Jia T, Zhang L, Duan Y, Zhang M, Wang G, Zhang J, et al. 2014. The differential susceptibilities of MCF-7 and MDA-MB231 cells to the cytotoxic effects of curcumin are associated with the PI3K/Akt-SKP2-Cip/Kips pathway. Cancer Cell Int. 14: 126.

35. Sui Q, Zhang J, Sun X, Zhang C, Han Q, Tian Z. 2014. NK cells are the crucial antitumor mediators when STAT3mediated immunosuppression is blocked in hepatocellular carcinoma. J. Immunol. 193: 2016-2023. 